\title{
Signal Enhancement in Surface Crack Detection with Gas-Coupled Laser Acoustic Detection
}

\author{
Dario Vangi ${ }^{1}$ - Mara Bruzzi ${ }^{2}$ James N. Caron ${ }^{3} \cdot$ Michelangelo S. Gulino $^{1}[$
}

Received: 19 March 2021 / Accepted: 8 September 2021 / Published online: 22 September 2021

(c) The Author(s) 2021

\begin{abstract}
Ultrasonic signal enhancement resulting from constructive interference between direct Rayleigh waves and same waves reflected by a surface defect is exploited to increase crack identification capabilities of the Gas-Coupled Laser Acoustic Detection (GCLAD) non-contact detection technology. Highlights from simulations are provided regarding the interference phenomenon in the solid and its propagation in air, where GCLAD detection occurs. Experimental campaigns are preliminarily performed on a bar to evidence the effect of cracks on the GCLAD acquired signals. Then, a signal enhancement of $+30 \%$ is reached on a plate, implying that defects are efficiently scanned by moving the GCLAD in proximity of the discontinuity. Since the GCLAD allows monitoring points of a piece belonging to the same line at once, its translation in one direction is sufficient to perform a two-dimensional scan, entailing reduction of inspection time and simple automation of the interrogation layout compared to other traditional or signal enhancement-based techniques.
\end{abstract}

Keywords Surface acoustic wave (SAW) - Non-contact detection $\cdot$ Constructive interference $\cdot$ Rayleigh wave $\cdot$ Line inspection · Plate inspection

\section{Introduction}

Surface acoustic waves (SAWs) are remarkably interesting for the non-destructive inspection of components and the detection of surface defects. In mechanical components where bending stresses are predominant like railway axles [1-4], surface defects represent the greatest criticality because of their propensity to propagate when subjected to fatigue states. Inspection by SAWs generally occurs in pulse-echo or pitch-catch mode. These inspection modes are typically eased by employing non-contact detection devices to rapidly scan extended surfaces. For instance, optical generation and detection of ultrasound respectively by pulsed lasers and interferometers are well-established and efficient

Michelangelo S. Gulino

michelangelosanto.gulino@unifi.it

1 Department of Industrial Engineering of Florence, Università degli Studi di Firenze, Via di Santa Marta 3, 50139 Florence, Italy

2 Department of Physics and Astronomy, Università degli Studi di Firenze, Via Sansone 1, 50019 Sesto Fiorentino, Italy

3 Research Support Instruments, 4325-B Forbes Boulevard, Lanham, MD 20706, USA
[5]; interferometers are however unsuitable to inspect poorly reflecting surfaces. Electro-Magnetic Acoustic Trasnsducers (EMATs) are a cost-efficient, compact alternative to optical devices. Still, the EMAT liftoff distance from the surface to be interrogated must be minimized to result in sufficient emitted/received ultrasonic amplitude (unfeasible in case of limited accessibility to the component); additionally, EMATs are applicable to conductive materials only and a proper optimization of their constitutive elements is required to counter their native transduction inefficiency [6-11]. In contrast, AirCoupled Transducers (ACTs) can be applied to the inspection of whichever material, but generation and detection of the waves occurs in air with significant energy loss along the propagation path $[12,13]$.

Inspection by these devices often involves scanning the surface of the component by moving both emitter and detector, with appropriate mutual spacing, along one direction. However, for defects far below the wavelength of the incident SAW, a significant part of the wave energy overcomes the defect. In both pulse-echo and pitch-catch mode, the defect is difficult to detect [14]. This drawback is accentuated when the distance between receiver and emitter increases, so that such distance should be limited. The use of higher frequencies, while reducing the wavelength and the size 
of the smallest detectable defect, increases the attenuation of the wave and consequently reduces the employable distance between emitter and receiver. Inspecting an extended region generally requires multiple scans, moving the system in the two orthogonal directions $x$ and $y$. Inspection by guided waves represents an exception to such rule, allowing defect detection in arbitrary positions even without moving the source or the detector; nevertheless, this typically applies to low thickness pieces like pipes or thin plates, in which SAWs typically travels a long path without significant attenuation $[15,16]$.

When inspection by SAWs is applied to a specimen in which a surface defect is present, constructive interference occurs at specific locations between the direct SAW and the same wave reflected by the crack flank, independently from the employed type of source or receiver. A signal enhancement effect is hence triggered, which favours identification of defects which are much smaller than the employed acoustic wavelength $\lambda[14,17]$. The signal enhancement effect represents a well-established concept in the non-contact ultrasonic inspection literature, especially for microcrack identification purposes. Kromine et al. [18,19] first highlighted the phenomenon employing a pulsed laser for excitation and an interferometer for detection, providing a first example of the Scanning Laser Source (SLS) technique where the defect is scanned by the emitter (i.e., the emitter is moved in the defect proximity); a piezoelectric contact probe was also employed for the reception of Rayleigh and Lamb waves with $\lambda=0.6$ $\mathrm{mm}$ to identify surface cracks with a depth ranging between $0.06 \mathrm{~mm}$ and $11 \mathrm{~mm}$, as well as a notch on a turbine disk. While Kromine et al. [19] reported an enhancement factor of two in correspondence of the crack, Blackshire and Sathish [20] subsequently reached an enhancement factor of four in case of Rayleigh wave excitation with $\lambda=0.3 \mathrm{~mm}$ by a piezoelectric contact probe and identification of defects with a depth down to $0.25 \mathrm{~mm}$. From that moment, several analyses followed employing different strategies to exploit the signal enhancement effect: Boonsang and Dewhurst [21] first employed an EMAT as a receiver of Rayleigh waves to detect a $0.5 \mathrm{~mm}$ long and $1.5 \mathrm{~mm}$ deep normal surface slot $(\lambda=2.6 \mathrm{~mm})$; Arias and Achenbach [17] introduced the concept of Scanning Laser Line Source (SLLS) in which the source laser spot of a typical SLS is focused on a line: by this solution, interaction between the thermally-induced elastic field and a finite length defect can be thoroughly studied by schematically referring to a simple 2D problem. Using the SLLS approach, Sohn and Krishnaswamy [22] detected 0.5 $\mathrm{mm}$ wide and $2.5 \mathrm{~mm}$ deep normal surface slots by $\lambda=0.3$ mm Rayleigh waves, with an amplification factor of 2.5. In several campaigns, Edwards et al. [23,24] employed EMATs as both receivers and emitters to detect $2.5 \mathrm{~mm}$ deep surface slots by Rayleigh waves with $\lambda=5.3 \mathrm{~mm}$, moving the receiver over the defect.
Using the SLLS in a configuration in which source and receiver are inverted, Dutton et al. [25] demonstrated that scanning the crack by the source is unbeneficial for the identification of angled defects: contribution of surface skimming longitudinal waves (SSLWs), generated by mode conversions in the proximity of angled defects, adds to constructive interference and solely if the source is close to the discontinuity; in fact, SSLWs sustain significant attenuation in a narrow path and do not typically reach the detector with significant energy, should the latter be placed far from the crack. Dutton et al. [25] reached an enhancement factor of approximately 12 in case of a defect inclined of $30^{\circ}$ with respect to the surface, identifying $2 \mathrm{~mm}$ deep and $0.5 \mathrm{~mm}$ wide cracks by Rayleigh waves with $\lambda=2.2 \mathrm{~mm}$. In the study by [26], a pulsed laser was employed as a source and moved over the defect, while a focused ACT monitored the propagating Lamb waves with $\lambda=16 \mathrm{~mm}$, enhanced by a $0.5 \mathrm{~mm}$ deep surface breaking fatigue crack. Rosli et al. [27] then detected $1 \mathrm{~mm}$ deep and 1 mm wide angled defects by the same method, while Zhou and $\mathrm{Xu}$ [14] evidenced $0.1 \mathrm{~mm}$ deep normal surface defects by Lamb waves with $\lambda=6 \mathrm{~mm}$ scanning the defect by the source. In two separate analyses, Trushkevych and Edwards [28,29] finally demonstrated that normal surface cracks or thermal fatigue cracks with a depth comprised between $0.5 \mathrm{~mm}$ and $2 \mathrm{~mm}$ can be evidenced by Rayleigh or Lamb waves, both in coated and uncoated samples. Based on the reported literature review, it is hence derived that no in-depth analysis exists on non-contact detection in air of enhanced Rayleigh waves, and more specifically considering that the defect should be preferably scanned by the receiver rather than the emitter (as suggested by $[25,30])$. Still, as will be demonstrated, refraction of interference-enhanced waves from a solid results in peculiar ultrasonic patterns in air, involving a high detection efficiency only for limited values of the detector liftoff distance from the specimen surface.

One example of optical methods for non-contact detection of ultrasonic waves is represented by Gas-Coupled Laser Acoustic Detection (GCLAD) [31,32]. The GCLAD technique is based on the displacement and deflection of a laser beam intersecting fluid regions with refractive index variations, which are caused by the refraction of the ultrasonic wave in the fluid itself (primarily air). The overall deviation of the GCLAD probe laser beam is detected by a positionsensitive photodetector $[33,34]$. This technique is relatively simple from the required instrumentation standpoint, has the advantage of being negligibly influenced by the finishing or reflectance of the component's surface and demonstrates a sensitivity comparable to a Fabry-Perot confocal interferometer [35]. Even if the characteristics of the technique and the suitability for defect detection have been recently demonstrated through the analysis of various experimental configurations by $[36,37]$, the GCLAD device currently fea- 
tures a low signal-to-noise ratio which limits its application range from an industrial perspective.

The technique can be employed in various arrangements of the laser beam with respect to the SAW propagating in the component, also depending on the geometry of the piece [36]. Some layouts for the laser beam with respect to the component allow a line inspection, i.e., to detect whichever defect present along a complete line of the piece without the need to perform a scan with the source/receiver along that line. This peculiarity makes the approach potentially advantageous compared to techniques where an $x-y$ scan of the entire surface is required, as only scanning along one direction of the piece is needed. In addition, exploitation of the signal enhancement effect is potentially beneficial to contextually increase the response of the GCLAD, enabling augmented sensitivity to surface defects.

This work aims to demonstrate the potential and limits of the GCLAD technique for the inspection of plane components with wide surfaces, e.g., employable for the monitoring of bars, plates or axisymmetric components (like axles or shafts). The GCLAD detector is moved in proximity of the defect, coherently with the indications by [25,30]; enhanced Rayleigh waves are detected in air after propagation in a steel bar and plate, in which artificial surface defects have been machined. Sensitivity of the technique for defect detection is highlighted, as a function of crack position and dimension. A brief overview of the system's operating principle and features is initially provided. Then, the materials and methods for the inspection of steel bars and plates are introduced and the inspection results subsequently highlighted; in the process, simulation results are also presented highlighting the currently unexplored translation of the signal enhancement effect from a solid to a fluid domain, where GCLAD detection is performed. Section 5 finally remarks the novel elements of the research based on the obtained results.

\section{GCLAD Technique}

The probe beam deflection or Gas-Coupled Laser Acoustic Detection (GCLAD) technique [34,38] relies on the deflection and displacement sustained by a laser beam while travelling into a fluid domain, based on the variation of the refractive index present in the same fluid. Such a variation can be associated with an acoustic wave propagating in the fluid, which determines a variable pressure field and a consequent variation in the refractive index [39]. A position-sensitive photodetector detects the displacement and/or deflection of the laser beam caused by the pressure field and converts those elements into a deviation-proportional voltage [38]. The position-sensitive photodetector is constituted of two physically separated cells, which are differently radiated by the beam based on the displacement it sustains; at each

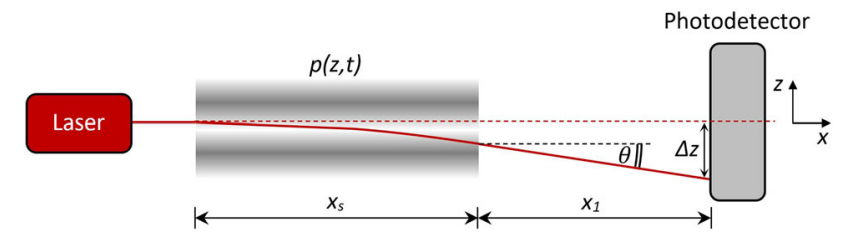

Fig. 1 A continuous wave modulable laser emits a beam which is initially displaced ( $x_{s}$ length) and then deflected $\left(x_{1}\right.$ length) by the variable pressure field $p(z, t)$. The overall displacement $\Delta z$ along the wave propagation direction $(z)$ is detected by a position-sensitive photodetector

instant, the radiation energy difference between the two cells provides the signal outputted by the detector.

Let us consider a sinusoidal pressure field generated by an acoustic wave propagating along the $z$ direction, starting from a component/fluid interface:

$p(z, t)=k \rho_{0} c^{2} \delta \sin (k z-\omega t) e^{-\alpha z}$

where $\omega$ represents the angular frequency of the acoustic wave, $c$ its speed in the fluid, $k$ its wave number $\omega / c, \alpha$ its attenuation coefficient in the fluid, $\rho_{0}$ the density of the unperturbed fluid, $z$ the distance from the component's surface (which represents the condition $z=0$ ), and $\delta$ the surface displacement (generating the ultrasound in the fluid). Referring to Fig. 1, starting from the eikonal equation [40], the displacement $\Delta z$ in correspondence of the photodiode for an infinitely small optical beam (propagating along $x$ perpendicularly to the planar pressure wave of Eq. 1) is obtained:

$$
\Delta z=\frac{2 \pi^{2}\left(n_{0}-1\right)}{c^{2} n_{0}} \delta f^{2} x_{s}^{2} \cos \left(k z_{0}-k c t\right)+\theta x_{1}
$$

where $n_{0}$ is the refractive index of the unperturbed fluid, $z_{0}$ the $z$ position of the unperturbed laser beam, $f$ the main frequency of the acoustic wave and $\theta$ the deflection angle (i.e., $\left.\theta=\frac{d(\Delta z)}{d x_{s}}\right)$. Hence, in this classical configuration, sensitivity for an optical beam $\Delta z / \delta$ increases as several parameters are increased, i.e., the length of the acoustic pressure field $x_{s}$ to which the laser beam is subjected, the distance from the photodiode $x_{1}$ and the acoustic wave frequency $f$. The expression is valid in the situation where the width of the optical beam is negligible compared to the acoustic wavelength. If not, the finite beam size reverses the behavior: a higher response for high acoustic wavelengths is observed, the beam diameter being the same [41]. As a non-contact detection device, GCLAD can sense variations in the refractive index associated with ultrasound induced by different types of contact or non-contact sources, like continuous wave lasers [42-46] or contact piezoelectric probes [37].

To obtain a response which is independent of the distance $x_{1}$, it is possible to employ the arrangement highlighted by [33] (Fig. 2) in which a focusing lens is placed before the 


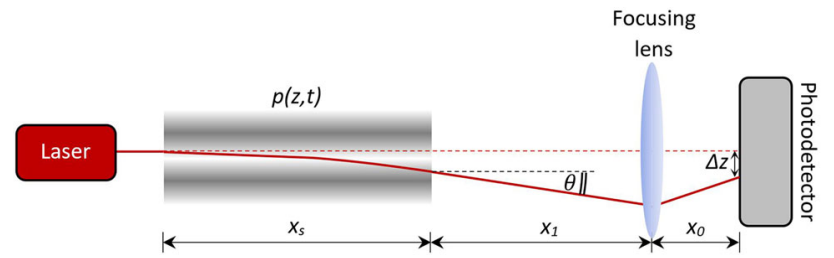

Fig. 2 GCLAD arrangement with a focusing lens placed at a distance $x_{0}$ from the photodetector, to make the system response independent of the $x_{1}$ length

photodetector. In such manner, it is possible to actually nullify the influence of deflection sustained by the beam on the detected signal; by this configuration, indicating as $x_{0}$ the distance between the focusing lens and the photodetector, the response is in fact [39]:

$\Delta z=\theta x_{0}$

Performance analysis for the system can undoubtly benefit from the configuration in Fig. 2, enabling isolation of the sole deflection contribution and making the system response more comparable among different experimental layouts. If not otherwise specified, the results reported in the following are obtained with the GCLAD configuration in Fig. 1 (no focusing lens).

\section{Materials and Methods}

The GCLAD system has been applied to observe the signal enhancement effect caused by interaction of Rayleigh waves with defects in the far-field. Specifically, a piezoelectric contact probe is placed on an inclined plexiglass wedge so that only Rayleigh waves can be excited inside steel specimens (a bar and a plate). These waves are subsequently refracted along their path from the solid surface to the fluid domain, represented by air; since the thickness of the samples more than doubles the acoustic wavelength in the solid, Lamb waves are not triggered inside the pieces.

The experimental arrangement for investigation on a bar is illustrated in Fig. 3. The laser beam is translated along the positive and negative direction of the $x$ axis, the origin of which is fixed at the centre of the defect. Since interaction between cracks and ultrasound is significantly affected by the mutual orientation between crack flank and wave propagation direction [47], tests have been performed as the angle $\beta$ between the bar and the direction of the GCLAD laser beam is varied. The laser beam is arranged parallel to the surface of the bar, at a variable height $d$ (liftoff distance). The distance between the laser and the photodetector is $85 \mathrm{~mm}$, while the centre of the bar is placed $14 \mathrm{~cm}$ away from the beam exit of the laser diode. The test piece is an S355 structural steel, $12 \mathrm{~mm}$ thick bar, with dimensions $520 \times 70 \mathrm{~mm}^{2}$. The defect consists of a $1 \mathrm{~mm}$ thick machined cut with an elliptical section, with a minor semi-axis (depth) of $3 \mathrm{~mm}$ and a major axis (length) of $20 \mathrm{~mm}$; the width of the crack (separation between its side surfaces) is equal to $1 \mathrm{~mm}$.

The GCLAD technique has been subsequently applied to scan a plate on which artificial defects have been machined. The plate is $12 \mathrm{~mm}$ thick, $500 \times 400 \mathrm{~mm}^{2}$ in size, and made of S355 structural steel. Four artificial defects have been triggered in the positions indicated in Fig. 4. The defects are nominally identical to those machined on the bar. For each $y$ position of the piezoelectric probe, a single scan has been performed in the $x$ direction by the laser probe beam.

The ultrasound is excited by a Panametrics contact piezoelectric probe with a frequency of $500 \mathrm{kHz}$ and a $25.4 \mathrm{~mm}$
Fig. 3 Experimental layout for the observation of the signal enhancement effect caused by the interaction between Rayleigh waves and surface defect on a bar, by moving the GCLAD detector in the defect proximity. Rayleigh waves are in this case obtained by a piezoelectric contact probe mounted on an angled plexiglass wedge support; the configuration allows analysing the effect of the mutual orientation $\beta$ between crack flank and Rayleigh wave propagation direction in the solid
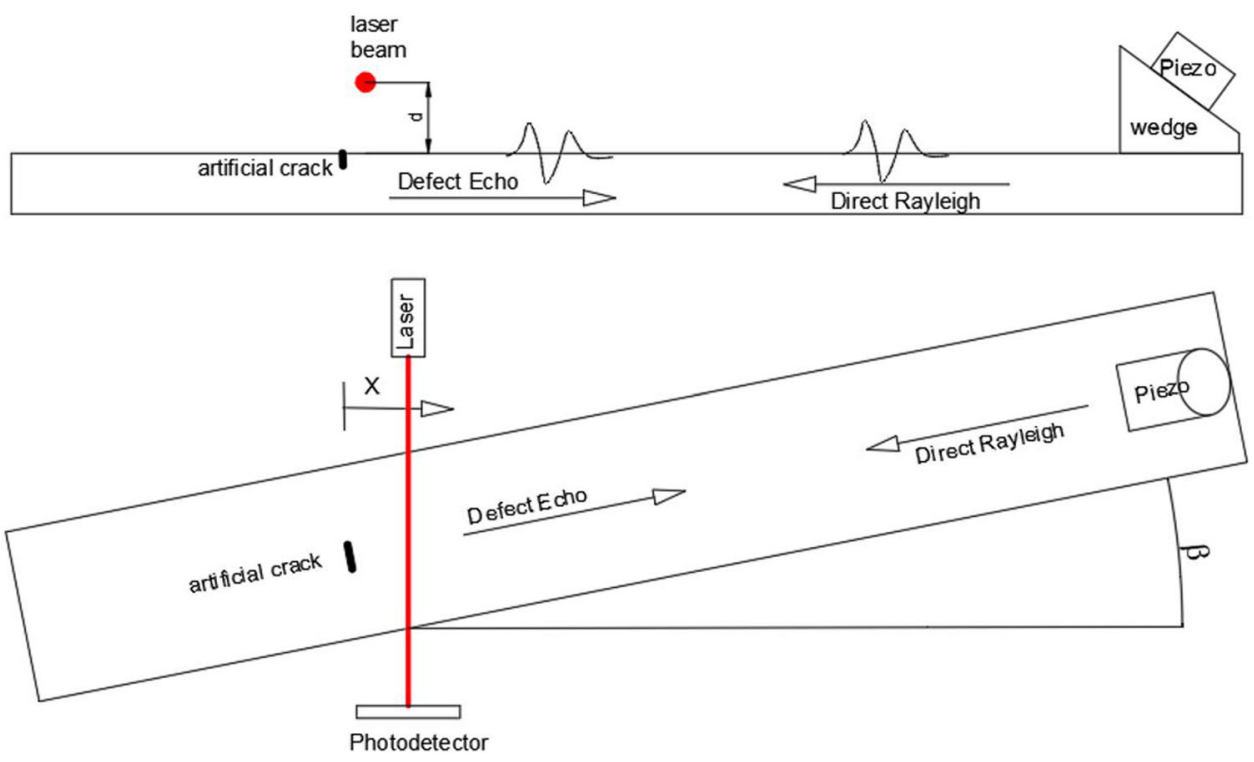


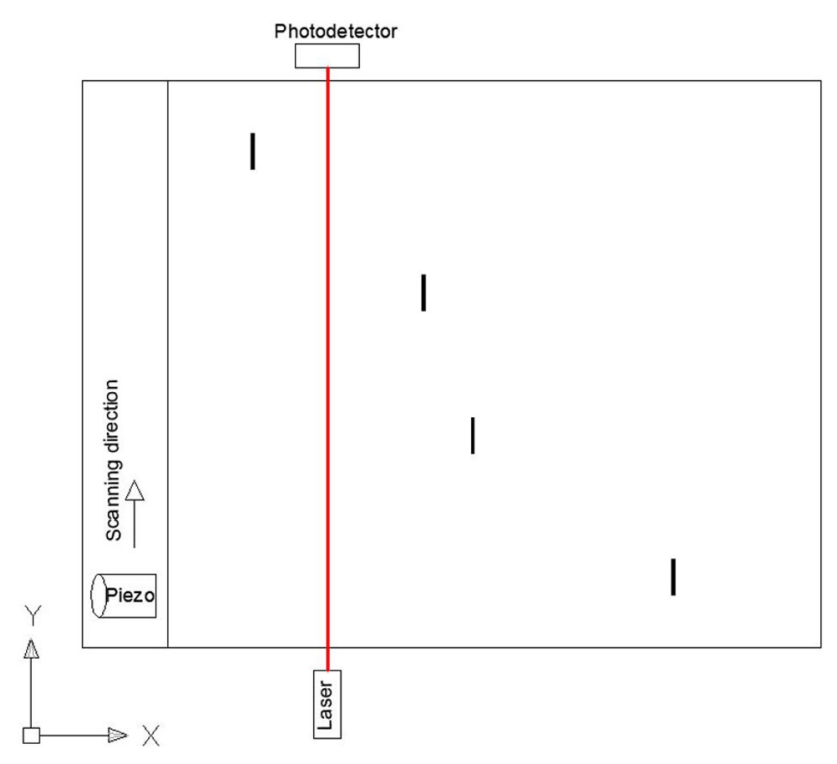

Fig. 4 Plate with positions of four artificial defects; the GCLAD device translates along the $x$ axis, while the piezoelectric contact probe is moved along the $y$ axis

diameter, with a $600 \mathrm{kHz}$ bandwidth. For the excitation of Rayleigh waves, a Panametrics plexiglass wedge support is employed. The Rayleigh wavelength in steel is about $5.7 \mathrm{~mm}$, higher than the depth of the artificial defects created in the test pieces. The laser employed is a TOPTICA iBeam Smart 640 with a power of $2 \mathrm{~mW}$, wavelength equal to $640 \mathrm{~nm}$, and beam at the exit of the device collimated on a $1.2 \times 0.7 \mathrm{~mm}^{2}$ elliptical spot (measured by a beam profiler). The employed photodiode for the GCLAD photodetector is a Red Enhanced Quad Cell Silicon Photodiode (SD 197-23-21-014) developed by Luna Optoelectronics. The signal acquisition from the photodiode is synchronized with the excitation of the piezoelectric probe. The signal hence obtained is pre-processed with a Brüel \& Kjær 2638 wideband signal conditioning. The filter is a bandpass filter from $300 \mathrm{kHz}$ to $2 \mathrm{MHz}$.

In Fig. 5, the experimental layout for plate inspection is highlighted. The plate is unpainted; nonetheless, sensitivity of the GCLAD device would not be affected by the painting or coating presence: ultrasonic detection occurs in air, so that the optical properties of the surface would not influence the response as for detection by other optical devices [35,36,41]. In this case, however, attenuation by the coating or painting stratum would necessarily result in a decrease in the ultrasonic wave amplitude [28]. No information is available on how the system would perform in different environments, for instance considering weather influence; currently, even if signal amplitudes are comparable to those from an ACT [37], additional optimizations from an electronics and a sensitivity standpoint are required for the GCLAD to become as reliable as other non-contact detectors like interferome-

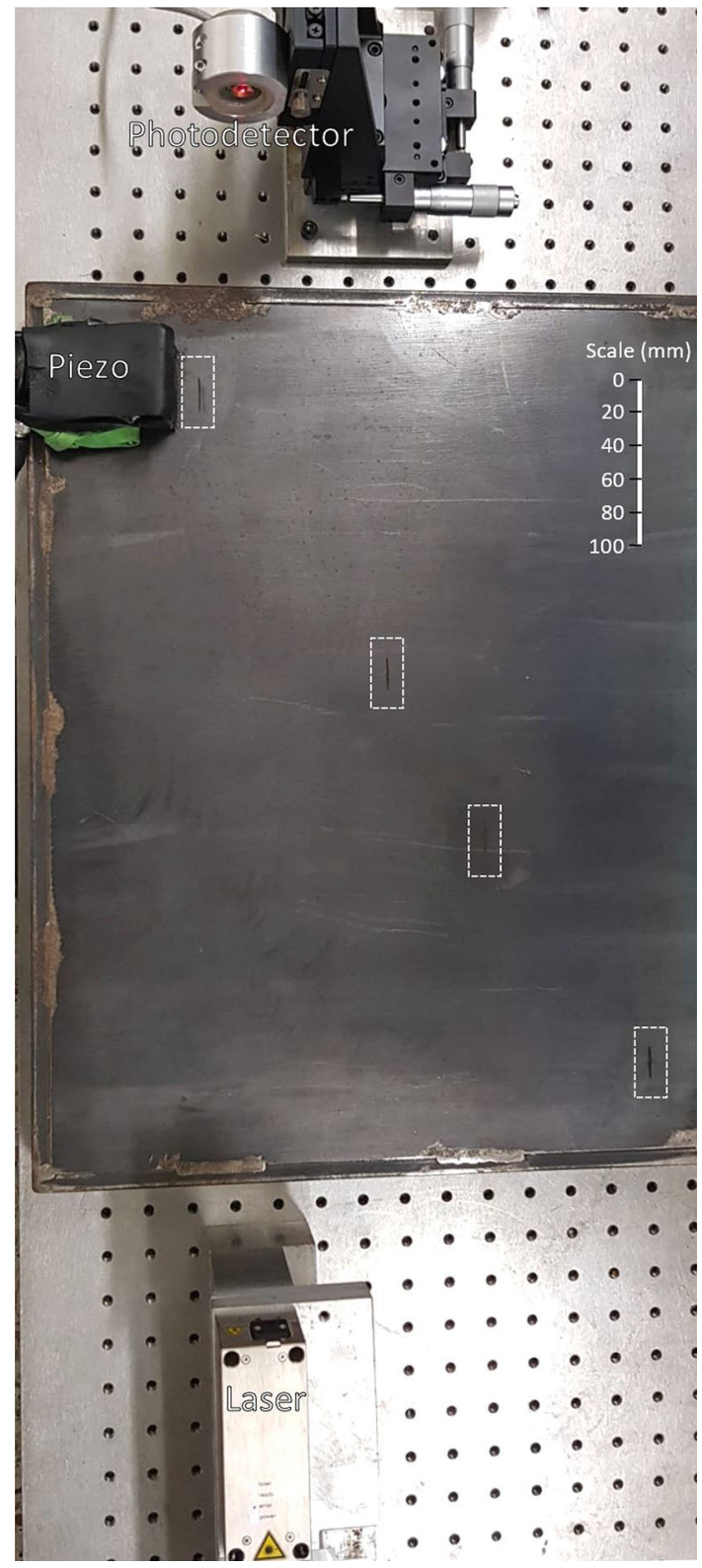

Fig. 5 Experimental layout for the steel plate inspection; the dashed rectangles represent locations of the artificial cracks

ters for inspection practices: the low signal-to-noise ratio is currently the main factor preventing GCLAD application to industrialized contexts.

\section{Signal Enhancement}

The results of tests performed to verify the signal enhancement effect by the GCLAD in the defect proximity (caused by the interaction of Rayleigh waves with the defect itself) and to assess performances in plate inspection tasks are separately provided. 
Fig. 6 Signal acquired in the position $x=80 \mathrm{~mm}$ with $\beta=0$
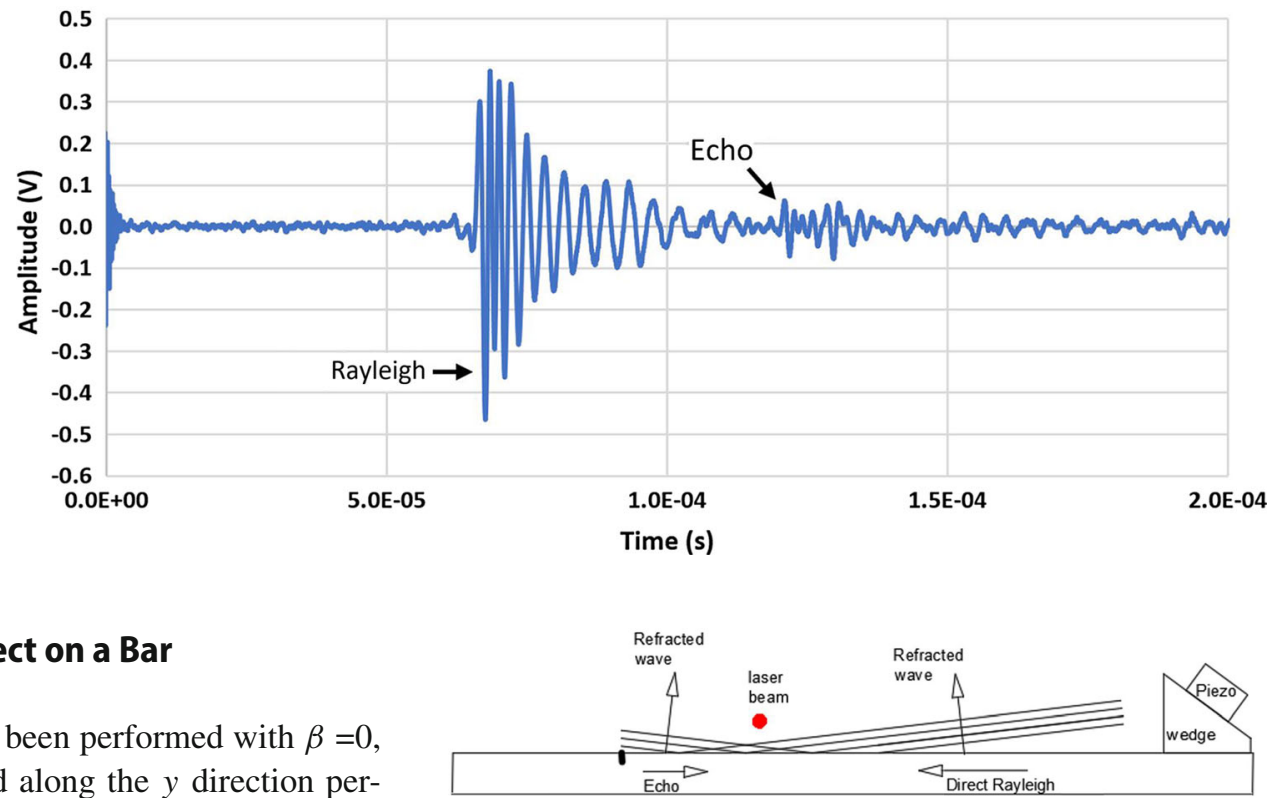

Initially, the tests of Fig. 3 have been performed with $\beta=0$, i.e., with the laser beam aligned along the $y$ direction perpendicular to the Rayleigh waves aligned along $x$ (emitted by the piezoelectric probe). The defect is aligned along the $y$ direction of the laser beam. In this case, the echo caused by the interaction of the Rayleigh waves with the defect also reflects in the direction perpendicular to the laser beam. Figure 6 shows a signal detected at the position $x=80 \mathrm{~mm}$. When the laser beam is far from the defect, the first received wave pattern relates to the direct Rayleigh waves generated by the piezoelectric probe; the echo is detected later and, as the defect approaches, it is mixed with the direct wave pattern.

The wave emitted by the piezoelectric probe and the wave reflected by the defect are both refracted in air, with an angle of 6.8 and -6.8 respectively, as depicted in Fig. 7.

The refracted waves overlap creating zones of constructive and destructive interference, varying in both time and space. Figure 8 shows a simulation of Rayleigh wave interaction with the discontinuity, in which the fluid (air) region of constructive and destructive interference near the defect is visible. The simulation duplicates the layout of Fig. 7 for the 2D solution of the visco-elastic linear wave propagation problem. The Wave $2000^{\odot 1}$ special-purpose software is used to simulate the propagation of longitudinal waves in the plexiglass wedge, whose mode varies at the interface with the metal component giving rise to Rayleigh waves. The wave excited by the probe consists of an exponentially damped pulse: in five periods, the amplitude of the 500 $\mathrm{kHz}$ oscillation is reduced to $10 \%$ of its maximum value. Rayleigh waves propagate until the defect is reached, which is overcome only by a minor part of the vibrational energy (low amplitude): this is because the crack depth is slightly lower than the ultrasonic wavelength. The first Rayleigh wave

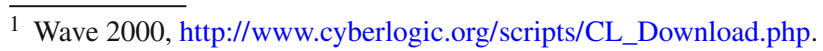

Fig. 7 Scheme of direct waves and waves reflected by the defect, refracted in air

oscillations reflected by the defect interfere with subsequent oscillations, first constructively (generating the B zone with high ultrasonic amplitude near the defect) and then destructively (low amplitude zone, A). The ultrasonic amplitude in the B zone decreases as the distance from the component's surface increases. While the related literature comprises indepth studies regarding the signal enhancement phenomenon as it occurs in the solid, the present analysis demonstrates that such an effect also transmits from a solid domain to the surrounding fluid; this allows highlighting and exploiting the signal enhancement effect even when ultrasonic detection is performed in air rather than in the solid. The retrieved information regarding the simulated waveforms additionally enables proper interpretation of signals obtained in experimental campaigns. For instance, considering the B zone alone, it is expected that the amplitude of the waves will be high only for a limited liftoff $d$ of the GCLAD laser beam with respect to the solid surface: over a $d$ value of $3 \mathrm{~mm}$, oscillations in air feature limited amplitude, even in the constructive interference zone.

Moving the GCLAD laser beam near the defect, it is therefore expected that the amplitude of the signal will change. Figure 9 shows the trend in the signal peak-to-peak amplitude received at the photodetector in the experimental campaign, as a function of the $x$ coordinate for various distances $d$ of the laser beam from the bar surface. In particular, the signal part analysed is that relating to the first packet of received waves (direct Rayleigh wave). Approaching the defect (decreasing positive $x$ ), the signal amplitude slightly decreases first. This is caused by the fact that the Rayleigh wave scatters while 
Fig. 8 Wave $2000^{\odot}$ simulation of the experimental layout in Fig. 7: the $500 \mathrm{kHz}$ probe excites a longitudinal wave in the plexiglass, which changes the mode at the interface with the metal component. The resulting Rayleigh wave reaches the crack and generates regions with low (destructive interference, A), high (constructive interference, $\mathrm{B}$ ), very low (in correspondence of the crack, C) and again low (crack overcome, D) ultrasonic amplitude

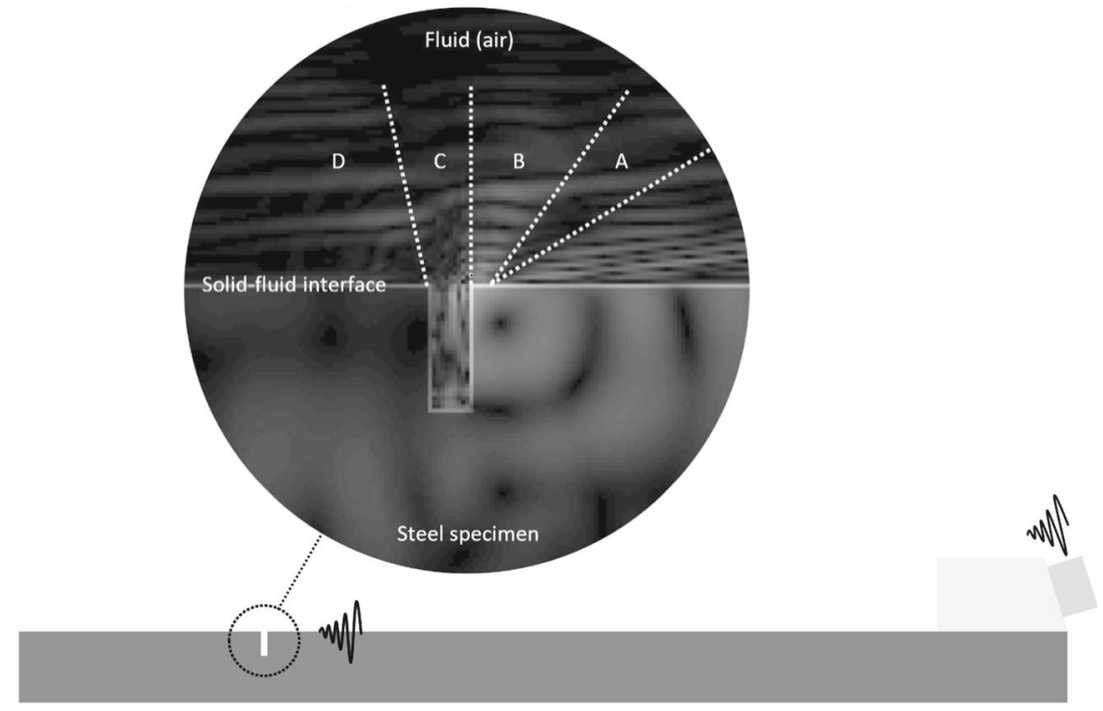

This phenomenon cannot be highlighted by traditional detection devices, like contact or air-coupled probes: the effect involves an extremely limited metal-air region near the defect, specifically with a width equal to half the ultrasonic wavelength (the low amplitude zone is generated from destructive interference between ultrasound at the same frequency, but with opposite phase). For such reason, it is necessary to rely on punctual transduction methods like interferometric sensors or, as in this case, GCLAD; if elements wider than the acoustic wavelength (such as traditional probes) are employed, oscillations in low and high amplitude zones are mediated so that information loss results.

The signal enhancement effect is attenuated as the inclination of the defect with respect to the GCLAD laser beam increases, i.e., as the angle $\beta$ increases. Figure 10 shows the trend in the peak-to-peak amplitude of the signal as a function of both $\beta$ and the distance $x$ from the defect, measured by placing the laser beam at a liftoff distance of $3 \mathrm{~mm}$ from the piece surface. This phenomenon is caused by two diverse instances:

a for $x=0$, the laser beam intersects the defect at its midpoint, and because the defect is unaligned with the beam direction, part of the beam is in the high amplitude region and another part is in the low amplitude region. The shift of the laser beam in correspondence of the photodetector, based on Eq. 2, is the integral of all movements sustained along its path. An averaging effect is hence observed;

b when the defect is angled with respect to the laser beam direction, the latter no longer lies on the plane of the echo wavefront in air; the beam can subsequently intersect multiple wavefronts. Again, the averaging effect caused by the integral of the displacements sustained by the beam along its path reduces the total displacement: the pressure gradient encountered by the beam varies, also reversing them refers to ultrasonic detection in air. 
Fig. 10 Trend in the peak-to-peak amplitude as a function of the $\beta$ angle and the $x$ distance between defect and laser beam

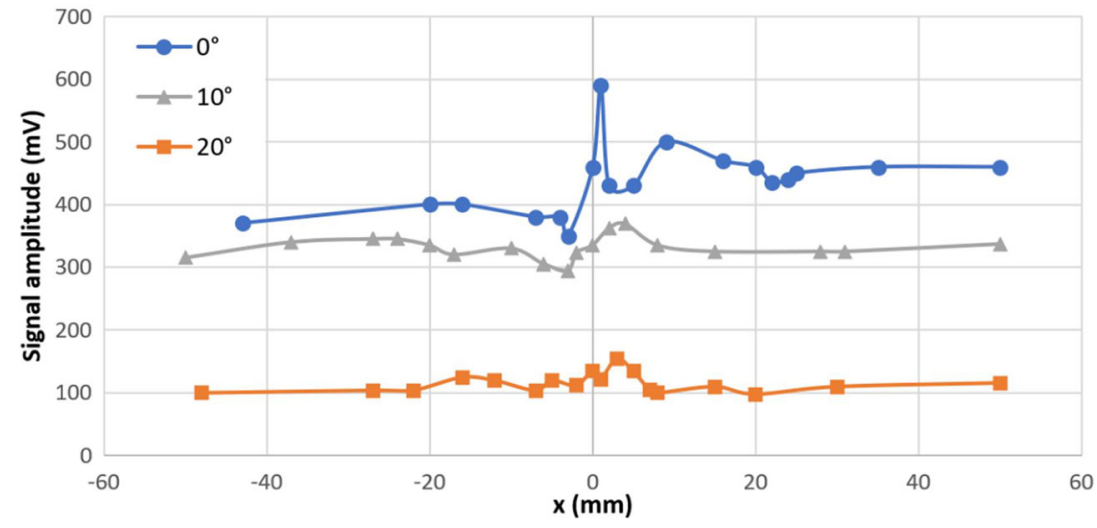

in sign as it completely crosses a period of the wave, according to Eq. 2 .

In Fig. 11, the trend in the peak-to-peak amplitude is reported, obtained when the laser beam is placed at $x=1$ $\mathrm{mm}$ as a function of $\beta$. The laser beam distance from the piece surface is $3 \mathrm{~mm}$. When a $\beta$ angle higher than $45^{\circ}$ is reached, the signal-to-noise ratio gets close to 1 and the signal cannot be distinguished from noise. The decrease in the signal amplitude occurs because of the two above reported mechanisms (a) and (b).

In Fig. 12, the trend in the peak-to-peak amplitude for the signal received positioning the laser at $x=80 \mathrm{~mm}$ is reported, as a function of the $\beta$ angle. In this case, the reduction in the signal amplitude as $\beta$ increases depends on the b) mechanism only. At such distance from the defect, the echo from the defect is also identifiable (Fig. 6), whose attenuation as the $\beta$ angle varies is also reported in Fig. 12. It is evident that the echo amplitude is similar to that of the direct signal, apart from a scaling factor. Such an overview demonstrates that defects can be detected also in case of non-orthogonal orientation between the crack flank and the Rayleigh wave propagation direction; the above cited effects a) and b) however limit crack identification to values of $\beta$ ranging between $-45^{\circ}$ and $45^{\circ}$.

\subsection{B-Scan of a Plate}

The signal enhancement effect can be advantageously employed for defect detection as it allows observing sudden amplitude variations of the first detected wavefront while the receiver position changes, instead of looking for defect-related echoes [14]; the defect echoes, in fact, often hide among spurious echoes associated with other wave paths within the component. If the distance between source and receiver is high, the signal amplitude is comparable to the original signal without defect even with the classical pitch-catch technique (considering the attenuation of waves in the material). Hence, small defects are not easily detectable by the traditional pitch-catch

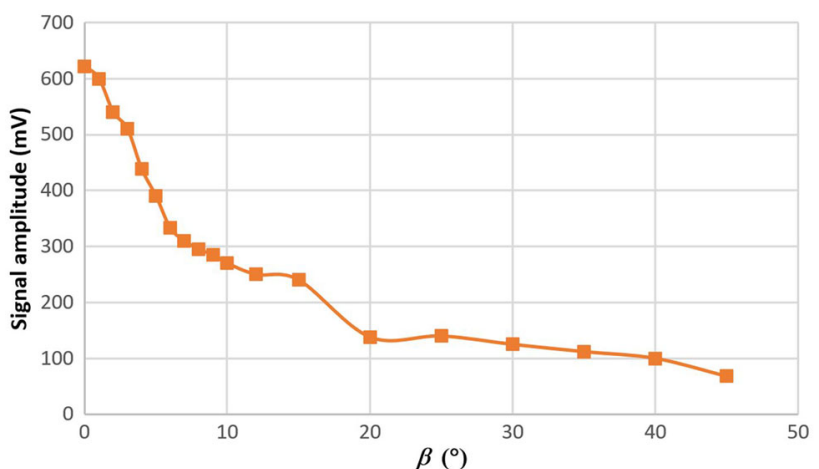

Fig. 11 Trend in the GCLAD amplitude acquired for $x=1 \mathrm{~mm}$ as a function of the $\beta$ angle

mode nor the pulse-echo mode. The signal enhancement effect, conversely, allows effectively detecting defects whose depth is even lesser than the wavelength of the Rayleigh wave [17].

As an example of application of the GCLAD technique, a plate has been scanned according to the scheme in Fig. 4. Figure 13 shows the $\mathrm{B}$-scan of the plate, where the peak-topeak amplitude is reported for each $x-y$ coordinate of the first wave pattern received by the photodetector. The piezoelectric contact probe has been positioned at the $y=0 \mathrm{~mm}$ coordinate, while the GCLAD has been moved along the $x$ direction with a step of $1 \mathrm{~mm}$, acquiring the signal at each translation. At the final $x$ coordinate $(x=400 \mathrm{~mm})$, the piezoelectric probe has been moved with a step of $5 \mathrm{~mm}$ in the positive $y$ direction and the procedure has been repeated moving the GCLAD device. Iterations have been performed until all $x$ and $y$ coordinates have been covered by the GCLAD device and the piezoelectric probe, respectively. Variations in amplitude when the laser beam position corresponds to the defect allow clearly highlighting the presence of the defect itself. In particular, the signal intensification immediately before the defect and the sudden reduction after the defect are both evident; the highest signal enhancement factor obtained in correspondence of the crack is equal to 1.3 for $\lambda=6.2 \mathrm{~mm}$ (as in the case of 


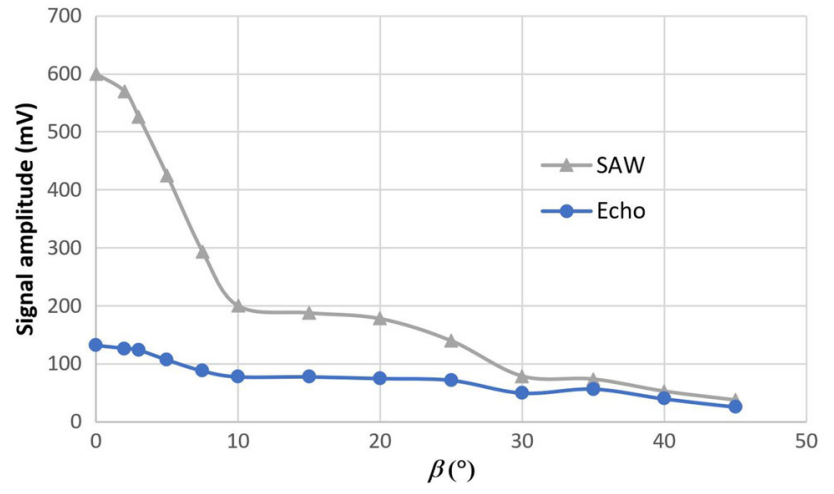

Fig. 12 Trend in the GCLAD amplitude of both direct wave and echo for $x=80 \mathrm{~mm}$ as a function of the $\beta$ angle

bar inspection), for the defect positioned at the maximum $y$ coordinate.

Although the defects are nominally the same, the variation in signal amplitude is different depending on the position of the defect in the plate. This is caused by the fact that each position of the defect corresponds, in the adopted scanning scheme, to a different value of $x_{1}$ (see Fig. 4); therefore, based on Eq. 2, a different laser beam displacement value in correspondence of the photodetector is associated with each crack position. It is observed that the value of $x_{s}$, which also influences the response of the GCLAD system based on Eq. 2 , remains unchanged for the various defects: the laser beam portion affected by the air-refracted echo wave has always the same width, equal to about the defect width $(20 \mathrm{~mm})$.

To obtain a response of the GCLAD which is independent of the defect position, the layout with a focusing lens shown in Fig. 2 can be employed. Figure 14 shows the result obtained by scanning the plate introducing a focusing lens inside the GCLAD layout. From a comparison between Figs. 13 and 14, it is highlighted that exclusion of the displacement contribution by the lens modifies the signal amplitude, lowering the maximum signal enhancement factor towards a value of $1.2(\lambda=6.2 \mathrm{~mm})$.

The evidenced signal enhancement factors are in line with those reported on aluminium plates by [28], who employed EMATs for both emission and detection of Rayleigh waves and scanned the defect by the detector; from this standpoint, detection in air rather than in the solid does not appear to affect the signal enhancement factor, but a higher crack depth has been here analysed ( $3 \mathrm{~mm}$ instead of $1.5 \mathrm{~mm}$ ) as well as a lower $\lambda(6.2 \mathrm{~mm}$ instead of $15 \mathrm{~mm}$ ). Referring once again to the considered crack depth, it is similar to that investigated by [22] (by a lower acoustic wavelength of $0.3 \mathrm{~mm}$ ); still, it does not reach the levels of the cracks explored by $[19,20,28]$, for instance down to $0.5 \mathrm{~mm}$ deep with a $\lambda$ of $8 \mathrm{~mm}$ [29]. Dhital and Lee [26] also found that almost closed cracks being 0.02 $\mathrm{mm}$ wide can be identified by a pulsed laser-focused ACT apparatus, in which enhanced Lamb waves with $\lambda=16 \mathrm{~mm}$ could be detected by overcoming the defect with the emitter.

Even if a lower wavelength has been considered in the present study and defects have reduced length compared to those identified by $[22,23,25]$, the aim is not to devise a solution featuring superior crack identification performances compared to other signal enhancement-based techniques. The objective is to widen the application range of the GCLAD technique, which is currently unestablished in industrial contexts because of the limited signal-to-noise ratios; based on the provided experimental evidences, it is demonstrated that exploitation of the signal enhancement phenomenon can be beneficial to increase sensitivity of the GCLAD device: surface cracks can be efficiently identified in the scanning process of components mainly extending in two dimensions, with an enhancement factor ranging between 1.2 and 1.3 for an acoustic wavelength equal to $6.2 \mathrm{~mm}$. In addition, time and automation required for the inspection are reduced compared to other signal enhancement-based methodologies: the GCLAD device allows inspecting all points on a line at once, requiring to move the system in a single direction only to perform an $x-y$ scan. Finally, it has been for the first time demonstrated that the signal enhancement effect can be highlighted also in air if the defect is scanned by the detector rather than the source, as suggested by $[30,48]$.

\section{Conclusions}

The present work highlights how constructive interference phenomena among direct and reflected Rayleigh waves in the vicinity of surface defects can be employed to increase the response of the Gas-Coupled Laser Acoustic Detection (GCLAD) device. This effect can be suitably employed for the identification of surface defects on a mechanical component which mainly extends in two dimensions, like metal plates and axisymmetric elements: by a numerical approach, it has been qualitatively demonstrated that constructive interference between direct and reflected Rayleigh wavefronts propagating on the piece surface also translates in air, domain in which GCLAD detection occurs. An increase in the amplitude of the ultrasound refracted in air equal to $30 \%$ compared to the case of direct wave has been experimentally estimated near the defect for a wavelength of $6.2 \mathrm{~mm}$. By continuously monitoring the changes in the amplitude of the direct wave, rather than searching for defect echoes often mixed with other ultrasonic paths inside the component, it is hence possible to unambiguously identify the position of the discontinuity. Since the GCLAD device enables line detection of waves propagating in the piece, its employment in a $2 \mathrm{D}$ monitoring process entails its movement along a single direction; decrease in inspection time and simplification of the interrogation layout are hence expected compared to tradi- 
Fig. 13 Results from the plate configuration in Fig. 1 (no focusing lens); variations in the evidenced in proximity of the defects $x-y$ scanning using the GCLAD peak-to-peak amplitude are

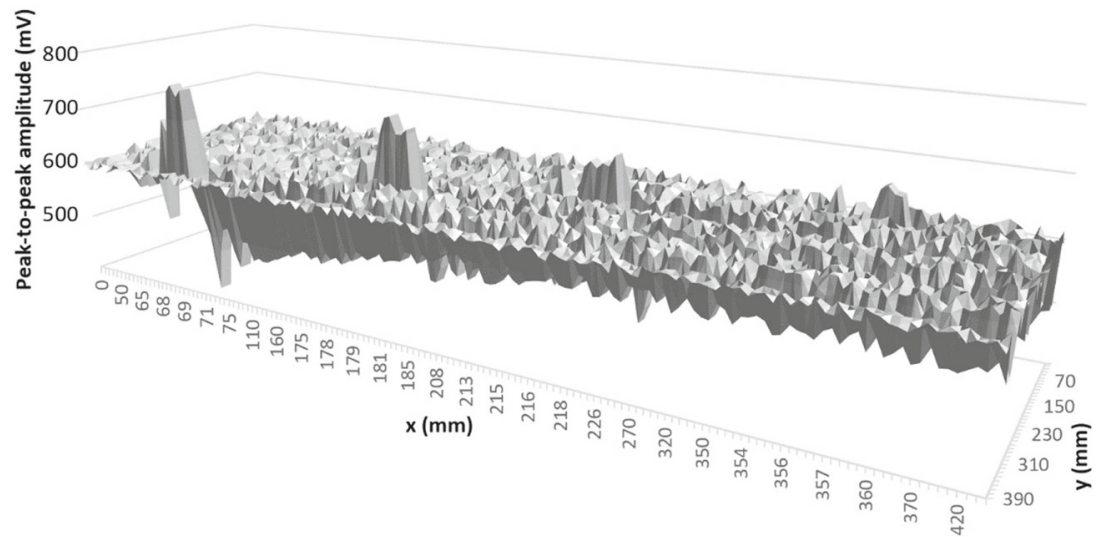

Fig. 14 Results from the plate $x-y$ scanning using the GCLAD configuration of Fig. 2 (focusing lens positioned before the photodetector); variations in the peak-to-peak amplitude with similar modulus are evidenced in proximity of the defects

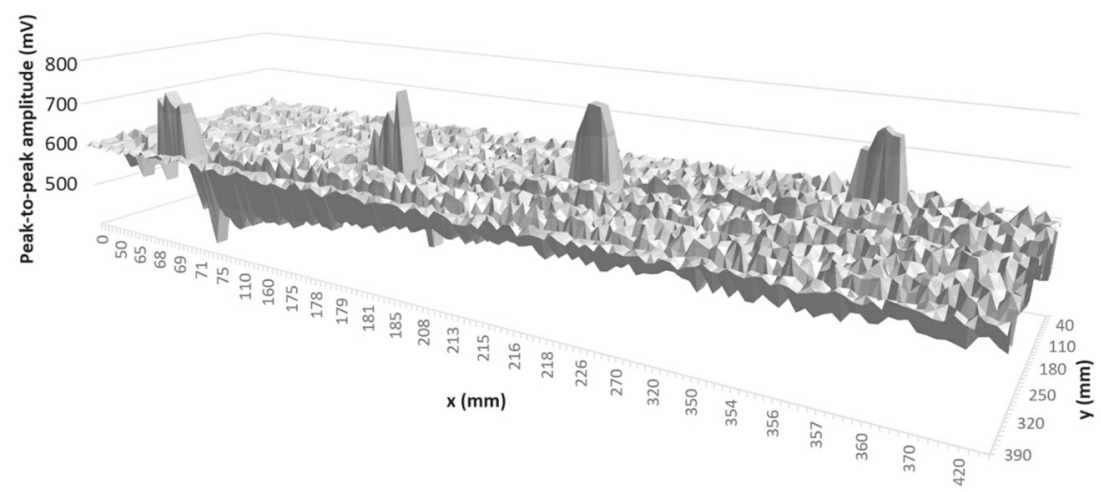

tional pitch-catch or pulse-echo modes, as well as other signal enhancement-based methodologies.

Signal enhancement factors similar to those from the available literature have been retrieved, as well as coherent dimensions for the identified cracks. While allowing $3 \mathrm{~mm}$ deep cracks to be highlighted in plates, the system is sensitive to the mutual orientation between defect surface and propagation direction of the GCLAD laser beam: the signal enhancement effect cannot be highlighted when angles above $45^{\circ}$ are reached. The study also demonstrates that scanning the defect by moving the detector (rather than the source) in positions close to the defects allows for crack identification, employing Rayleigh waves that are detected after refraction from the solid into the air.

The GCLAD technique represents a valid alternative for the detection of cracks through the proposed methodology, on components primarily extending in two dimensions; this is reflected in the possibility to both increase the automation of the inspection compared to classic contact sensors and leverage completely different physical principles than those exploited by air-coupled probes, paving the way for new NDT monitoring methodologies.

Acknowledgements The work has been performed as part of the STILUS project (Sviluppo di Tecniche Innovative di controllo nondistruttivo mediante Laser-Ultrasuoni per la Sicurezza degli assili fer- roviari), supported by Fondazione CR Firenzehttps://www.fondazionecrfirenze. it/.

Funding Open access funding provided by Università degli Studi di Firenze within the CRUI-CARE Agreement.

\section{Declarations}

Conflict of interest The authors declare that they have no conflict of interest.

Open Access This article is licensed under a Creative Commons Attribution 4.0 International License, which permits use, sharing, adaptation, distribution and reproduction in any medium or format, as long as you give appropriate credit to the original author(s) and the source, provide a link to the Creative Commons licence, and indicate if changes were made. The images or other third party material in this article are included in the article's Creative Commons licence, unless indicated otherwise in a credit line to the material. If material is not included in the article's Creative Commons licence and your intended use is not permitted by statutory regulation or exceeds the permitted use, you will need to obtain permission directly from the copyright holder. To view a copy of this licence, visit http://creativecomm ons.org/licenses/by/4.0/. 


\section{References}

1. Montinaro, N., Epasto, G., Cerniglia, D., Guglielmino, E.: Laser ultrasonics for defect evaluation on coated railway axles. NDT \& E Int. 116, 102321 (2020). https://doi.org/10.1016/j.ndteint.2020. 102321

2. Cavuto, A., Martarelli, M., Pandarese, G., Revel, G., Tomasini, E.: Experimental investigation by laser ultrasonics for high speed train axle diagnostics. Ultrasonics 55, 48-57 (2015). https://doi.org/10. 1016/j.ultras.2014.08.010

3. Cavuto, A., Martarelli, M., Pandarese, G., Revel, G., Tomasini, E.: FEM based design of experiment for train wheelset diagnostics by laser ultrasonics. Ultrasonics 113, 106368 (2021). https://doi.org/ 10.1016/j.ultras.2021.106368

4. Mineo, C., Cerniglia, D., Pantano, A.: Numerical study for a new methodology of flaws detection in train axles. Ultrasonics 54(3), 841-849 (2014). https://doi.org/10.1016/j.ultras.2013.10.008

5. Scruby, C.B., Drain, L.E.: Laser Ultrasonics: Techniques and Applications. A. Hilger, Bristol (1990)

6. Thring, C., Fan, Y., Edwards, R.: Multi-coil focused EMAT for characterisation of surface-breaking defects of arbitrary orientation. NDT \& E Int. 88, 1-7 (2017). https://doi.org/10.1016/j. ndteint.2017.02.005

7. Xie, S., Tian, M., Xiao, P., Pei, C., Chen, Z., Takagi, T.: A hybrid nondestructive testing method of pulsed eddy current testing and electromagnetic acoustic transducer techniques for simultaneous surface and volumetric defects inspection. NDT \& E Int. 86, 153 163 (2017). https://doi.org/10.1016/j.ndteint.2016.12.006

8. Tkocz, J., Greenshields, D., Dixon, S.: High power phased EMAT arrays for nondestructive testing of as-cast steel. NDT \& E Int. 102, 47-55 (2019). https://doi.org/10.1016/j.ndteint.2018.11.001

9. Liu, Z., Deng, L., Zhang, Y., Li, A., Bin, W., He, C.: Development of an omni-directional magnetic-concentrator-type electromagnetic acoustic transducer. NDT \& E Int. 109, 102193 (2020). https:// doi.org/10.1016/j.ndteint.2019.102193

10. Xiang, L., Edwards, R.S.: Multiple wavemode scanning for near and far-side defect characterisation. J. Nondestruct. Eval. 39(1), 9 (2020). https://doi.org/10.1007/s10921-019-0651-0

11. Augustyniak, M., Usarek, Z.: Finite element method applied in electromagnetic NDTE: a review. J. Nondestruct. Eval. 35(3), 39 (2016). https://doi.org/10.1007/s10921-016-0356-6

12. Garcia-Rodriguez, M., Yañez, Y., Garcia-Hernandez, M., Salazar, J., Turo, A., Chavez, J.: Application of Golay codes to improve the dynamic range in ultrasonic Lamb waves air-coupled systems. NDT \& E Int. 43(8), 677-686 (2010). https://doi.org/10.1016/j. ndteint.2010.07.005

13. Römmeler, A., Furrer, R., Sennhauser, U., Lübke, B., Wermelinger, J., de Agostini, A., Dual, J., Zolliker, P., Neuenschwander, J.: Air coupled ultrasonic defect detection in polymer pipes. NDT \& E Int. 102, 244-253 (2019). https://doi.org/10.1016/j.ndteint.2018. 12.004

14. Zhou, Zhang, $\mathrm{Xu}$, Ren: Defect detection of aluminium plates based on near-field enhancement of lamb waves generated using an electromagnetic acoustic transducer. Sensors 19(16), 3529 (2019). https://doi.org/10.3390/s19163529

15. Köhler, B., Kim, Y., Chwelatiuk, K., Tschöke, K., Schubert, F., Schubert, L.: A mode-switchable guided elastic wave transducer. J. Nondestruct. Eval. 39(2), 45 (2020). https://doi.org/10.1007/ s10921-020-00690-5

16. Cho, Y.: Model-based guided wave NDE: the evolution of guided wave NDE from "magic" to "physically based engineering tool". J. Nondestruct. Eval. 31(4), 324-338 (2012). https://doi.org/10. 1007/s10921-012-0151-y

17. Arias, I., Achenbach, J.D.: A model for the ultrasonic detection of surface-breaking cracks by the scanning laser source tech- nique. Wave Motion 39(1), 61-75 (2004). https://doi.org/10.1016/ j.wavemoti.2003.06.001

18. Kromine, A., Fomitchov, P., Krishnaswamy, S., Achenbach, J.: Laser ultrasonic detection of surface breaking discontinuities: scanning laser source technique. Mater. Eval. 58(2), 173-177 (2000a)

19. Kromine, A.K., Fomitchov, P.A., Krishnaswamy, S., Achenbach, J.D.: Applications of scanning laser source technique for detection of surface-breaking defects. In: Halliwell, N.A. (ed.) Symposium on Applied Photonics, Glasgow, UK, pp. 252-259 (2000b). https:// doi.org/10.1117/12.397958

20. Blackshire, J.L., Sathish, S.: Near-field ultrasonic scattering from surface-breaking cracks. Appl. Phys. Lett. 80(18), 3442-3444 (2002). https://doi.org/10.1063/1.1476722

21. Boonsang, S., Dewhurst, R.J.: Enhancement of laserultrasound/electromagnetic-acoustic transducer signals from Rayleigh wave interaction at surface features. Appl. Phys. Lett. 82(19), 3348-3350 (2003). https://doi.org/10.1063/1.1571980

22. Sohn, Y., Krishnaswamy, S.: Interaction of a scanning lasergenerated ultrasonic line source with a surface-breaking flaw. J. Acoust. Soc. Am. 115(1), 172-181 (2004). https://doi.org/10. $1121 / 1.1630997$

23. Edwards, R.S., Dixon, S., Jian, X.: Enhancement of the Rayleigh wave signal at surface defects. J. Phys. D Appl. Phys. 37(16), 22912297 (2004). https://doi.org/10.1088/0022-3727/37/16/011

24. Edwards, R.S., Jian, X., Fan, Y., Dixon, S.: Signal enhancement of the in-plane and out-of-plane Rayleigh wave components. Appl. Phys. Lett. 87(19), 194104 (2005). https://doi.org/10.1063/ 1.2128058

25. Dutton, B., Clough, A.R., Edwards, R.S.: Near field enhancements from angled surface defects; a comparison of scanning laser source and scanning laser detection techniques. J. Nondestruct. Eval. 30(2), 64-70 (2011a). https://doi.org/10.1007/s10921-0110091-y

26. Dhital, D., Lee, J.R.: A fully non-contact ultrasonic propagation imaging system for closed surface crack evaluation. Exp. Mech. 52(8), 1111-1122 (2012). https://doi.org/10.1007/s11340011-9567-z

27. Rosli, M., Edwards, R., Fan, Y.: In-plane and out-of-plane measurements of Rayleigh waves using EMATs for characterising surface cracks. NDT \& E Int. 49, 1-9 (2012). https://doi.org/10.1016/j. ndteint.2012.03.002

28. Trushkevych, O., Edwards, R.: Characterisation of small defects using miniaturised EMAT system. NDT \& E Int. 107, 102140 (2019). https://doi.org/10.1016/j.ndteint.2019.102140

29. Trushkevych, O., Edwards, R.S.: Differential coil EMAT for simultaneous detection of in-plane and out-of-plane components of surface acoustic waves. IEEE Sens. J. 20(19), 11156-11162 (2020). https://doi.org/10.1109/JSEN.2020.2996154

30. Hernandez-Valle, F., Edwards, R.S., Clough, A.R., Rosli, M.H., Dutton, B.: Scanning laser techniques for characterisation of different surface breaking defect geometries. In: Proceedings of 18th World Conference on Non-Destructive Testing, Durban, South Africa (2012)

31. Caron, J.N., Mehl, J.B., Steiner, K.V.: Ultrasonic NDE of composite panels with gas-coupled laser acoustic detection. In: Thompson, D.O., Chimenti, D.E. (eds.) Review of Progress in Quantitative Nondestructive Evaluation, pp. 635-642. Springer, Boston (1998a). https://doi.org/10.1007/978-1-4615-5339-7_82

32. Caron, J.N., Yang, Y., Mehl, J.B., Steiner, K.V.: Gas-coupled laser acoustic detection at ultrasonic and audio frequencies. Rev. Sci. Instrum. 69(8), 2912-2917 (1998b). https://doi.org/10.1063/ 1.1149033

33. Caron, J.N., Kunapareddy, P.: Application of gas-coupled laser acoustic detection to gelatins and underwater sensing. In: 40th Annual Review of Progress in Quantitative Nondestructive Evaluation: Incorporating the 10th International Conference on 
Barkhausen Noise and Micromagnetic Testing, Baltimore, MD, USA, pp. 458-463 (2014) https://doi.org/10.1063/1.4864855

34. Barnes, R.A., Maswadi, S., Glickman, R., Shadaram, M.: Probe beam deflection technique as acoustic emission directionality sensor with photoacoustic emission source. Appl. Opt. 53(3), 511 (2014). https://doi.org/10.1364/AO.53.000511

35. Caron, J.N., Thompson, D.O., Chimenti, D.E.: Displacement and deflection of an optical beam by airborne ultrasound. In: AIP Conference Proceedings, AIP, Golden, vol. 975, pp. 247-254 (2008). https://doi.org/10.1063/1.2902666

36. Gulino, M.S., Bruzzi, M., Vangi, D.: Gas-coupled laser acoustic detection technique for NDT of mechanical components. Ultrasonics 114, 106415 (2021). https://doi.org/10.1016/j.ultras.2021. 106415

37. Vangi, D., Bruzzi, M., Caron, J.N., Gulino, M.S.: Crack detection with gas-coupled laser acoustic detection technique. Meas. Sci. Technol. (2021b). https://doi.org/10.1088/1361-6501/abfced

38. Caron, J.N.: Displacement and deflection sensitivity of gas-coupled laser acoustic detection. In: 1st International Symposium on Laser Ultrasonics: Science, Technology and Applications, pp. 1-6 (2008)

39. Johnson, J.L., van Wijk, K., Caron, J.N., Timmerman, M.: Gascoupled laser acoustic detection as a non-contact line detector for photoacoustic and ultrasound imaging. J. Opt. 18(2), 024005 (2016). https://doi.org/10.1088/2040-8978/18/2/024005

40. Yariv, A., et al.: Optical Electronics in Modern Communications, vol. 1. Oxford University Press, Oxford (1997)

41. Gulino, M.S., Bruzzi, M., Vangi, D.: Application of the gas-coupled laser acoustic detection technique to non-destructive testing of mechanical components. In: XXVIII AIVELA Conference (2020)

42. Caron, J.N., DiComo, G.P., Nikitin, S.: Continuous laser generation of ultrasound for nondestructive evaluation. In: Review of Progress in Quantitative Nondestructive Evaluation: vol. 31, Burlington, VT, pp. 243-250 (2012) .https://doi.org/10.1063/1.4716236

43. Vangi, D., Virga, A., Gulino, M.: Study on the most influential parameters in low-power laser generated ultrasound. FME Trans. 45(3), 323-330 (2017a). https://doi.org/10.5937/fmet1703323V

44. Vangi, D., Virga, A., Gulino, M.S.: Random sequence for optimal low-power laser generated ultrasound. J. Phys. Conf. Ser. 882, 012013 (2017b). https://doi.org/10.1088/1742-6596/882/1/ 012013
45. Vangi, D., Gulino, M.S., Virga, A.: Optimal Rayleigh waves generation by continuous wave modulated laser. J. Phys. Conf. Ser. 1110, 012006 (2018). https://doi.org/10.1088/1742-6596/1110/1/ 012006

46. Vangi, D., Banelli, L., Gulino, M.S.: Interference-based amplification for CW laser-induced photoacoustic signals. Ultrasonics $\mathbf{1 1 0}$, 106270 (2021a). https://doi.org/10.1016/j.ultras.2020.106270

47. Kemppainen, M., Virkkunen, I.: Crack characteristics and their importance to NDE. J. Nondestruct. Eval. 30(3), 143-157 (2011). https://doi.org/10.1007/s10921-011-0102-z

48. Dutton, B., Clough, A.R., Rosli, M.H., Edwards, R.S.: Exploring surface wave interaction with angled defects in the near and far field. J. Phys. Conf. Ser. 278, 012011 (2011b). https://doi.org/10. 1088/1742-6596/278/1/012011

Publisher's Note Springer Nature remains neutral with regard to jurisdictional claims in published maps and institutional affiliations. 